\title{
Metamorfoses da cultura: do impresso ao digital, criando novos formatos e papéis em ambientes de informação
}

\author{
Mirian de Albuquerque Aquino \\ Professora doutora do Departamento de Biblioteconomia e \\ Documentação e do Programa de Pós-graduação em Educação Popular, \\ Comunicação e Cultura da Universidade Federal da Paraíba \\ E-mail:miriabu@uol.com.br
}

\section{Resumo}

O presente texto recupera a trajetória da biblioteca em seus diferentes formatos, mostrando a importância desta instituição em vários contextos. Aborda a passagem da cultura impressa para a cultura digital e a necessidade de os profissionais da informação se adequarem aos novos formatos. A expansão da cultura e do saber exige a aquisição de novas competências e habilidades para o desenvolvimento dos serviços informacionais. As ferramentas de busca, a recuperação e as estratégias de acesso à informação possibilitam ao indivíduo um contato mais rápido e direto com o objeto do conhecimento em qualquer lugar e tempo real. A Biblioteca Digital Paulo Freire dissemina os conteúdos filosóficos, sociológicos e pedagógicos do pensamento freireano.

\section{Palavras-chave}

Cultura impressa; Cultura digital; Ambientes de informação; Profissionais da informação.

\section{Metamorphoses of culture: from the printed paper to the digital, creating new formats and papers in environments information}

\begin{abstract}
This paper tracks down the development of library in its different formats, showing the importance of this institution in several contexts. It describes the changes from printed to digital culture and the information professionals' need to adapt themselves to the new formats. The expansion of culture and knowledge demands the acquisition of new competencies and abilities for the development of new information services. The search tools, retrieval and strategies of access to information enable the individual to have a faster and more direct contact with the object of knowledge in any place and real time. Paulo Freire Digital Library disseminates the philosophical, sociological and pedagogical contents of Paulo Freire's works, spread out in Brazil and several other countries.
\end{abstract}

\section{Keywords}

Printed culture; Digital culture; Digital library; Paulo Freire.
Uma coisa é certa: vivemos hoje em uma destas épocas limitrofes na qual toda a antiga ordem das representações e dos saberes oscila para dar lugar a imaginários, modos de conhecimento e estilos da regulação sociais ainda pouco estabilizados (...) um novo estilo de humanidade é inventado.

(Lévy, 1996, p. 17)

\section{DO IMPRESSO AO DIGITAL: ACESSANDO MEMÓRIA, TEMPO E ESPAÇO}

A discussão da biblioteca como um ambiente digital e novo espaço de interação humana acena para a reconstituição da história da cultura impressa e do livro e para a indagação sobre como essa instituição social atravessou as agruras seculares para sobreviver até os dias atuais. É, de certa forma, a história do registro do conhecimento, ou seja, o processo de construção da linguagem, que é o segredo e a explicação do homem (Martins, 1998), que, no decorrer de sua existência material e espiritual, sentiu a necessidade de criar um ambiente que permitisse armazenar, organizar, controlar e recuperar as informações, impedindo sua dispersão. Implica ainda compreender a natureza dos materiais utilizados na construção dos acervos, os agentes de conservação e os conceitos de serviços e sua disponibilização nos diferentes períodos históricos.

Essa ancoragem demanda longa narração dos acontecimentos que marcaram a história das bibliotecas e que talvez permitam reconhecer a complexidade desse ambiente de informação, saber, conhecimento e cultura, sua transmutação por diferentes formatos e sua materialização, a qual se deu, inicialmente, por meio das tabletas de argila, passando pelo papiro, pelo pergaminho e pelo papel, até transformar o formato impresso no formato digital ou virtual. No entanto, não é objetivo desse texto passar pela mundivivência da biblioteca, mas apenas pontuar alguns episódios da passagem dessa instituição, sua importância e seu papel atual para os leitores.

Diversas fontes de informação registram que a biblioteca, em sua perspectiva de expansão, conseguiu fazer história pelo mundo e em todas as épocas, assinalando, por exemplo, que a Biblioteca da Assíria viveu o apogeu do 
reinado de Assurbanipal e que a Biblioteca de Alexandria foi considerada à época o maior acontecimento do mundo, destacando-se não só por ter ocupado o lugar de centro literário científico-cultural, mas também por assumir uma posição proeminente na cultura grega. $\mathrm{Na}$ incursão pela Biblioteca de Pérgamo, é possível acompanhar seu desenvolvimento no mundo antigo, onde assumiu a tarefa de desenvolver um projeto real que pretendia se transformar em um centro literário e lingüístico.

É possivel ainda acompanhar o desenvolvimento de bibliotecas públicas e particulares de personalidades famosas como Virgílio, Varrão, Quinto, Aurélio e outros. A sociedade grega, por sua vez, conheceu diversas iniciativas de construção de bibliotecas privadas, onde o luxo e a ostentação podiam ser observados tanto no aspecto físico quanto na localização do acervo que refletiam, quase sempre, os saques de guerras. Essas bibliotecas se notabilizaram pelos serviços prestados à comunidade como depositárias de válidos documentos públicos e também pela disseminação da cultura, emprestando as suas obras e incentivando a leitura. Por intermédio dessas bibliotecas, os intelectuais obtinham livros copiados pelos escribas.

É conveniente ressaltar que, na Idade Média, essas iniciativas não se vinculavam aos projetos das bibliotecas romanas. $\mathrm{Na}$ literatura especializada, é possivel observar uma coincidência relacionada ao caráter ideológico e à opulência que revestiam os projetos político-culturais dos medievais. No embate com a ideologia filosófica cristã, esta decretou a decadência da cultura pagã, de modo que grande parte dessas bibliotecas e suas valiosas coleções foram destruídas, provocando um caos cultural que passou a ser representado pelo número assustador de analfabetos.

Nesse contexto de turbulência, o discurso religioso voltou-se para a coleção de textos sagrados, sua leitura e interpretação, resultando na construção de bibliotecas em centros culturais renomados. Essa preocupação se estendeu por toda a Europa, onde os monges puderam exercer a tarefa de reproduzir os manuscritos necessários ao desempenho de suas funções, desenvolvendo acervos nas bibliotecas famosas e, em contrapartida, distanciando os leitores dos livros.

Na obra O Nome da Rosa, o crítico literário italiano Umberto Eco descreve a antiga biblioteca de um convento beneditino, onde se guardavam códices preciosos que se constituíam parte da renomada sabedoria grega e latina conservada pelos monges através dos séculos. Essa biblioteca nasceu de um formato obscuro e inacessível e permaneceu ausente do público durante muitos séculos, privando o leitor do uso da informação, porque essa biblioteca guardava um segredo que só poderia ser contado a um bibliotecário-auxiliar, antes que a morte surpreendesse o bibliotecário-chefe, que detinha o monopólio da informação. Somente este último podia percorrer o labirinto dos livros, encontrálos, guardá-los e os conservar; só ele sabia a ordem de colocação do volume, do grau de sua inacessibilidade, do tipo de segredo, das verdades ou das mentiras que o volume encerrava (Eco, 1983, p. 53).

A disponibilização da informação raramente ocorria no contexto medieval porquanto se considerava que nem todas as verdades produzidas pelos indivíduos poderiam ser conhecidas por outros e, menos ainda, as mentiras poderiam ser desmascaradas, de modo que um modelo de exclusão se estabelecia: os leitores deveriam ler alguns livros, e não outros, para não caírem na tentação ou insensata curiosidade de saber aquilo que os homens fazem. Essa biblioteca, segundo Eco, era um escrínio e, por isso, não podia ser penetrada por qualquer mortal.

A crítica barthesiana é importante nessa discussão, exigindo-nos uma reflexão sobre a biblioteca, principalmente na sociedade brasileira, porque ela ainda é um lugar que se visita, mas no qual pouco se habita. Nas bibliotecas públicas, universitárias ou especializadas, a ausência de livros atualizados e interessantes é tão impactante que inibe o desejo de ler.

Para Barthes (1987), a questão não trata de contestar a instituição bibliotecária nem de perdermos o interesse pelo desenvolvimento que necessita. Para ele, "trata-se, mera e simplesmente, de reconhecer seu recalcamento que existe nesse traço fundamental e inevitável da biblioteca, que é a sua artificialidade" (Barthes, 1987, p.34). Esse raciocínio barthesiano está umbilicalmente ligado ao formato da biblioteca convencional, vez que esse autor não teve o privilégio de presenciar a instauração da cultura digital em que a biblioteca parece ser inesgotável. No entanto, as suas palavras, muitas vezes, dão-nos a impressão de que ele já antevia o nascer de uma biblioteca infinita com uma dimensão que depende dos objetivos de cada leitor.

(...) Por estatuto, seja qual for a sua dimensão, é infinita $_{2}$ na medida em que ela está sempre aquém ou além da procura, pois tendencialmente, o livro desejado nunca está lá, ao passo que nos é proposto um outro livro: a biblioteca é o espaço dos substitutos de desejo: perante a aventura de ler, ela é o real, por chamar o desejo à ordem, sempre demasiada grande e demasiada pequena, ela é fundamentalmente 
inadequada ao desejo: para tirar prazer, satisfação, fruição de uma biblioteca, o sujeito tem de renunciar à efusão do seu imaginário (Barthes, 1987, p. 34).

O sonho de uma biblioteca que reúna todos os saberes acumulados atravessou a história da nossa civilização, desde a mítica Biblioteca de Alexandria, na Antigüidade Clássica, passando pelo projeto de Mallarmé, que há um século pensava criar um livro integral (o livro), infinito, síntese de todos os livros passados e por vir (Parente, 1999, p. 68). Um mesmo sonho repetido através dos séculos aparece hoje nos textos eletrônicos armazenados de forma digital nos computadores, disquetes, CD-ROMs, os quais, por não se fixarem em suportes materiais como o papel, permitem o seu acesso a distância, em tempo real, ou seja, instantaneamente.

As conexões da informática com a telemática têm sido responsáveis pelo surgimento da informação em diferentes formatos de acesso e uso dessa informação. A passagem da cultura impressa para a cultura digital afetou não só os ambientes do papel, exigindo-lhes não só sua adequação aos novos formatos, mas impondo a aquisição de novas competências e habilidades para o desenvolvimento dos serviços informacionais. Ao derrubar as paredes que isolavam a biblioteca de seus leitores, expandiram-se a cultura e o saber, acenando-se para novas ferramentas de busca, recuperação e estratégias de acesso à informação, possibilitando, assim, aos indivíduos, um contato mais rápido e direto com o objeto do conhecimento em qualquer lugar e tempo real.

As bibliotecas digitais transformam-se em portão de entrada para os recursos mundiais de informação, trazendo significativas implicações para usuários de bibliotecas, provedores de informação, pesquisadores de todas as áreas do conhecimento. Esses ambientes, hoje reconfigurados pela presença do computador, e as possibilidades oferecidas pela Internet passaram a requerer um usuário com uma formação que o capacite para ler, escrever interpretar sua realidade, expressar-se adequadamente, lidar com conceitos abstratos, trabalhar em grupos de resolução de problemas, tomar decisões individuais e coletivas e, principalmente, "aprender a aprender" (Brennand et alii, 2000).

Nessa afirmação da autora, depreendemos a compreensão de que as tecnologias da informação e comunicação estão desafiando a educação para se familiarizar com as novas ferramentas e redesenhar novas formas de aprendizagem, a partir das quais o conhecimento pode ser gerado, recuperado, acessado, disponibilizado e utilizado. Essa compreensão de aprendizagem é reafirmada nas metas do Programa Sociedade da Informação no Brasil - Livro Verde, que propõem o desenvolvimento de competências amplas para atuar efetivamente "na produção de bens e serviços, tomar decisões fundamentadas no conhecimento, operar com fluência novos meios e ferramentas em seu trabalho, bem como aplicar criativamente as novas mídias, seja em uso simples e rotineiro, seja em aplicações mais sofisticadas" (Takahaski, 2000, p.45).

O uso das tecnologias no campo da educação não tem sido freqüente, quando comparada a outros setores sociais, implicando um desconhecimento de sua importância na formulação e disseminação de projetos educacionais relevantes. Esses projetos poderiam fomentar a participação mais intensiva de grupos de ação local, uma vez que essas tecnologias rompem o controle das políticas educativas centralizadas, abrindo espaços em comunidades não atendidas pelas mesmas, facilitando o aumento do nível de instrução e de acesso a bens culturais (Brennand et alii, 2000).

Brennand enfatiza que professores, alunos, pesquisadores e bibliotecários na sociedade brasileira têm lidado com grande quantidade de informação no formato impresso, o que dificulta, muitas vezes, o acompanhamento da atualidade e velocidade das informações sobre uma determinada área do conhecimento. Essa realidade tecnológica impõe a disponibilização da informação em suporte digital, em que é possível fazer a junção de recursos mais dinâmicos, como música, animação, gráficos, fotografia, vídeo etc., que podem ser transportados em um computador comum para qualquer lugar, retocados, mudados, combinados, manipulados, melhorados, reconstruídos ou mesmos impressos, produzindo um tipo de informação interessante (Chen, 2000). Por meio do computador, a informação pode ser processada, transmitida, memorizada e disponibilizada.

A velocidade do processamento de conteúdos, as facilidades de armazenamento, a presença de multimídias e telecomunicações possibilitam o acesso aos recursos informacionais em todo o mundo; logo a visão tradicional do ambiente no qual a informação impressa é estocada e mantida localmente se desestabiliza, à medida que a informação digital circula na rede em qualquer espaço e em tempo real.

\section{O MODELO DIGITAL É UM DOS MEIOS DAS METAMORFOSES: FORMATOS E PAPÉIS}

As grandes tendências da evolução das técnicas contemporâneas vieram acompanhadas das mutações sociais e culturais geradas no final dos anos 80 e início dos anos 90, dando visibilidade a um movimento 
sociocultural de superação da invenção do computador pessoal que instaurou um desenvolvimento tecnoeconômico sem precedentes na história do conhecimento e da informação.

Para Lèvy, as tecnologias digitais surgem como a infraestrutura do ciberespaço, novo espaço de comunicação, de sociabilidade, de organização de transação e de novo mercado da informação e do conhecimento. Para ele, o desenvolvimento do digital é, portanto, "sistematizante e universalisante não apenas em si mesmo, mas também, em segundo plano, a serviço de outros fenômenos tecnossociais que tendem à integração mundial: finanças, comércio, pesquisa científica, mídias, transportes, produção industrial etc.” (Lèvy, 1999, p. 113). Digital é a existência imaterial das imagens, sons e textos definidos matematicamente e processados por algoritmos* na memória hipertextual do computador (Silva, 2000).

Essa discussão, porém, que envolve a cultura digital, supõe uma consideração sobre o termo digitalizar, que, segundo Lèvy (1999), consiste em traduzir uma informação em números. Essa possibilidade permite que todas as informações possam ser codificadas, de maneira que as letras, os textos, as imagens e os sons se tornem objetos de digitalização, de modo que cada unidade desses objetos corresponda a um número, sendo expresso por meio de um sistema conhecido como linguagem binária. As informações codificadas nessa linguagem são traduzidas como textos legíveis, imagens audíveis, sons audíveis, sensações tácteis (Lèvy, 1999), automaticamente, com um grau de precisão absoluto, rapidez e em grande escala quantitativa transmitida e copiada quase indefinidamente sem perda de informação. Uma grande variedade de dispositivos técnicos permite gravar e transmitir os números codificados nessa linguagem.

Para Lèvy, o digital é uma matéria pronta a suportar todas as metamorfoses, todos os revestimentos, todas as deformações. É como se o fluido numérico fosse composto por uma infinidade de pequenas membranas vibrantes, cada bit sendo uma interface, capaz de mudar o estado de um circuito, de passar do sim ao não, de acordo com as circunstâncias (Lèvy, 1999, p. 103).

Para Silva (2000, p. 74), o digital é o absoluto da montagem, incidindo esta sobre os mais ínfimos fragmentos da mensagem, uma disponibilidade indefinida e incessantemente reaberta à combinação, à mixagem,

\footnotetext{
* São "conceitos científicos operacionalizados como disposição para múltiplas intervenções-navegações da parte do usuário" (Silva, 2000, p.70).
}

ao reordenamento dos signos. A tecnologia digital autoriza a fabricação de mensagens, sua modificação, bit por bit. Essa cultura abarca a digitalização como um processo que engloba todas as técnicas de comunicação e processamento de informações (Lèvy, 1996, p. 102). Segundo o autor, "a digitalização penetrou primeiro na produção e gravação de músicas, mas os microprocessadores e as memórias digitais tendiam a tornar-se a infra-estrutura de produção de todo domínio de comunicação" (Lèvy, 1999, p. 32), conectando, no centro de um mesmo tecido digital, o cinema, a radiotelevisão, o jornalismo, a edição, a música, as telecomunicações e a informática.

O modelo digital, capaz de conectar, num mesmo tecido eletrônico, a imagem, o som e a escritura, e, com isso, capaz de conectar, dentro de sua rede [todas as outras mídias]. Por ser, em si mesmo, um princípio de interface, a codificação digital, com seus bits de imagens, textos, sons, imbrica, nas suas tramas, nosso pensamento e nossos sentidos. É o grande processador leve, móvel, maleável e inquebrantável (Santaella, 1997, p. 41).

Hoje, para ter acesso às informações, os usuários não precisam mais esperar por investimentos do governo ou pela boa vontade dos dirigentes das instituições, porque os textos, que vinham em qualquer formato, em vez de chegar às bibliotecas no fundo de um caminhão-baú, nos porões do navio ou no bagageiro do avião, "podem viajar diretamente em sua forma digital através de cabos de coxias de cobre, por fibras óticas ou por via hertziana” (Lévy, 1999, p. 35).

A educação precisa estar atenta ao estilo digital de apreensão do conhecimento, isto é, ao estilo de conhecimento engendrado pelas novas tecnologias, para se inserir nos novos espaços de aprendizagem, produção da leitura e do conhecimento. Nesse ponto, é válido reconhecer a contribuição que o educador Paulo Freire inseriu na educação brasileira, não só para atualizar as suas idéias por meio da cultura impressa, mas disponibilizá-la, reinventando, recriando e rescrevendo, a partir de vários suportes. Relendo as idéias de Freire, Gadotti capta a relação que demonstrava ter com as tecnologias na prática educativa.

Ele tinha verdadeiro prazer em aprender e transmitia esse prazer para os que conviviam com ele, fosse na sala de aula, fosse em outros lugares [...] Desde suas primeiras experiências no Nordeste brasileiro, no início dos anos 60, ele buscava fundamentar o ensinoaprendizagem em ambientes interativos, através do 
uso de recursos audiovisuais. Mais tarde reforçou o uso de novas tecnologias, principalmente o vídeo, a televisão e a informática. Mas não aceitava a sua utilização de forma acrítica (Gadotti, 1998, p. 30).

Para Freire, "é tão urgente quanto necessária à compreensão correta da tecnologia, a que recusa entendêla como obra diabólica ameaçando sempre os seres humanos, ou a que perfila como constantemente a serviço de seu bem-estar" (Freire, 2000, p. 100). É preciso impedir a tecnologia de falar por si mesma, para tomá-la mais como um espaço de aprendizagem.

Então, é justo que continuemos a não só reconhecer a importância da cultura midiática, mas também que insistamos na necessidade de nos familiarizar com ela, para sabermos usá-la nas atividades cotidianas. É fundamental atentarmos para o confronto de novos desafios que as inovações tecnológicas nos põem hoje, abrindo mais espaços para a liberdade de criar (Freire, 2000, p. 101). A sintonia de Freire com as tecnologias pode ser vista por meio das palavras de Gadotti:

Nos últimos anos, ele estava programando uma série de vídeos para possibilitar o acesso ao conhecimento a um maior número de pessoas. Não se aprende tudo na escola, continuava insistindo. A TV, o vídeo e o computador podem ser ferramentas preciosas para as camadas populares. Mas precisamos aprender a ser emissores, e não apenas receptores de idéias. Prezava a cultura midiática. Ela pode tanto ser um complemento do que aprendemos na escola como também um motor do conhecimento. [...] A mídia pode sensibilizar-nos e a escola pode fazer parte desta sensibilização para ir além (Gadotti, 1998, p. 31).

A cultura midiática "contém potencialidades de criação e de concretização de novas e diferentes formas de educar e de pensar" (Guattari apud Maraschin, 2000, p. 106). É talvez por essa via que Freire tenta avaliar o significado da atualização do modo de fazer o ensino. Essa atualização pode ser feita por meio de outras ferramentas de aprendizagem, isto é, a convivência com os espaços digitais e a relação do sujeito com as atividades mediadas por computador, com as formas culturais e o uso de diversas tecnologias associadas à Web, na sociedade da informação, sociedade da aprendizagem ou sociedade do conhecimento.

Freire percebeu a reestruturação tecnológica, as transformações culturais, as novas possibilidades de acesso à informação, a integração das tecnologias ao cotidiano e a geração de imagens.
Outro dado do momento atual nos contextos que sofrem impacto da modernização tecnológica é a exigência que se coloca de decisões rápidas e variadas a desafios inesperados [...] Me parece demasiado óbvio que a educação de que precisamos, capaz de formar pessoas críticas, de raciocínio rápido, com sentido de risco, curiosas, indagadoras, não pode ser a que exercita a memorização dos educandos (Freire, 2000, p. 100).

Essa percepção freireana foi compartilhada com todos aqueles e aquelas que lidam com a educação, para viver o seu tempo. Para Silva (2000, p. 70-71), urge a necessidade de a escola sintonizar-se com a emergência da interatividade, sair da defensiva e abandonar os rituais de transmissão, porque os alunos apresentam como "novos expectadores, quando aprendem a manipular imagens digitais nas telas cada vez mais movimentadas, supondo uma nova dimensão comunicacional".

A passagem da cultura impressa para a cultura midiática, própria das transformações culturais, substituiu as práticas tradicionais de educar, pensar o ensino e interagir com o conhecimento "por artefatos e ferramentas por dispositivos em múltiplas conexões de sistemas que envolvem modens, telefones, computadores, satélites, redes e outros inventos que auxiliam na produção e na comunicação" (Domingues, 1997, p. 18).

\section{BIBLIOTECA DIGITAL PAULO FREIRE (BDPF): UM RECIPIENTE DO CONTEÚDO FREIREANO}

Em seus escritos, Brennand et alii (2000) observam que uma grande parte do legado freireano ainda se encontra registrada na forma impressa, de maneira que esse conteúdo ainda é pouco conhecido do público diversificado. Essa constatação, sendo mais visível no campo da educação, alicerçou a concepção, o desenvolvimento e a implementação de uma biblioteca digital que tem como principal objetivo disseminar conteúdos temáticos referenciados ao legado filosófico, sociológico e pedagógico do educador Paulo Freire e sua decisiva contribuição para a sociedade brasileira e a mundial.

A gênese dessa biblioteca, que constitui um ambiente de informação, está relacionada à compreensão de que a antiga ordem de representações das coisas e dos saberes está abrindo espaço para novos projetos, novos modos de conhecimento e cultura. Esse ambiente se contextualiza numa contemporaneidade tecnológica, intensamente marcada pelas recentes transformações materializadas na reorganização do trabalho, produção de negócios, bens e serviços e modificando as relações 
do indivíduo com a ciência, a tecnologia, o trabalho e os instrumentos necessários a essa atividade.

A criação dessa biblioteca sintoniza-se com as estratégias propostas no Programa Sociedade da Informação, criado pelo Ministério de Ciência e Tecnologia, e concebido para preparar novas gerações de redes com vistas a atender às demandas do desenvolvimento social e econômico do país, como parte do Plano Plurianual 2000-2004. O plano se estrutura em oito linhas de ação, incluindo projetos de educação a distância, criação e difusão cultural, criação de bibliotecas digitais, no sentido de colocar o mundo virtual como habilitador de competências e de participação social (Brennand et alii, 2000).

Para a autora, as metas educacionais veiculadas nesse programa englobam a interação com os meios e os serviços disponibilizados pelas tecnologias da informação e comunicação, colocando em evidência a perspectiva de treinamento e formação tecnológica com vistas à popularização da cultura digital. Em uma de suas linhas de atuação, tal programa incentiva a criação de bibliotecas digitais como forma de disseminação da informação, pela possibilidade de produção, tratamento de conteúdos, recuperação de informações e disponibilização on-line de conteúdos de interesse educacional e social (Brennand et alii, 2000).

$\mathrm{Na}$ criação dessa biblioteca, enfatiza a autora, consideraram-se, também, os seguintes aspectos relevantes: a) as orientações do Programa de Informação para a Pesquisa (Prossiga), que investe na criação de novos serviços de informação para a pesquisa, ou seja, a criação de bibliotecas virtuais na Internet; b) a relevância concedida à educação por parte das instituições governamentais, de maneira que o Programa da Sociedade de Informação venha coadunar com os objetivos de ampliação da ação educativa nos projetos educativos Vivendo e Aprendendo e Pedagogia da Pergunta, do Programa Ação Cultural, desenvolvidos pela UFPB e UFPE.

A proposta de criação dessa biblioteca visa a fortalecer a ampliação dos espaços de difusão de informações e atender à expansão do item qualidade nas pesquisas e nos projetos desenvolvidos nas instituições proponentes (Brennand et alii, 2000). Iniciada em 2000 e consolidada em 2003 como um projeto dinâmico, desenvolvido em parceria com a UFPB, a UFPE e o Centro de Estudos e Pesquisas Paulo Freire, essa biblioteca se vincula às linhas de pesquisa Estudos Culturais e Tecnologias da Informação e Comunicação do Programa de Pós- graduação em Educação e ao Grupo de Pesquisa Informação, Cidadania e Memória do Curso de Mestrado em Ciência da Informação da UFPB, tendo o apoio do Departamento de Informática e a Coordenação Institucional de Educação a Distância (Cead). Nesse projeto estão engajados três estudantes do Programa Institucional de Bolsa de Iniciação Científica (Pibic/ $\mathrm{CNPq} / \mathrm{UFPB}$ ), quatro bolsistas vinculados ao Projeto Institucional de Desenvolvimento de Métodos e Técnicas Inovadoras do Ensino, financiado pelo Sesu/MEC/ UFPB, cinco mestrandos do Programa de Pós-graduação em Educação Popular, Informação e Cultura, cinco bibliotecários e dois representantes do Núcleo de Tecnologia da Informação da UFPB.

A Biblioteca Digital Paulo Freire se constitui um referencial de pesquisa que permite ser acessado a qualquer hora e em qualquer lugar, tornando-se um espaço democrático que propicia o acesso, sem restrições, de todo material informativo necessário à educação e à aprendizagem, em todos os níveis de ensino. Trata-se de uma construção coletiva, implicando ações pontuais, as quais se fazem presentes, também, no pensamento freireano, podendo ser utilizadas na formação e no fortalecimento do trabalho cooperativo. Segundo Freire, "é na pluralidade de relações que os indivíduos estabelecem relações uns com os outros, que vão reconstruindo e redirecionando a capacidade de organizar as melhores respostas aos múltiplos desafios. Ao testar e agir, isto é, pela práxis, os indivíduos constroem sua consciência crítica" (Brennand et alii, 2000, p. 9).

Essa biblioteca tem como principal objetivo conceber, implementar e disponibilizar conteúdos relacionados aos pressupostos filosóficos, epistemológicos, sociológicos e pedagógicos de Paulo Freire, com a perspectiva de dar suporte a ações educativas democráticas que tenham como vetor o desenvolvimento de competências de participação social, facilitando a inserção dos sujeitos educativos na sociedade de informação (Brennand et alii, 2000).

Do ponto de vista conceitual, a literatura especializada sugere diversos modos de apreender as bibliotecas digitais. De modo geral, afirma York (2002, p. 1), a Digital Library Federation (DLF) concebe-as como "organizações que fornecem os recursos, incluindo o pessoal especializado, para selecionar, estruturar, oferecer acesso intelectual, traduzir, distribuir, preservar a integridade e garantir a permanência das coleções digitais, de tal forma que elas estejam disponíveis para uma ou várias comunidades". Uma biblioteca digital, segundo Cunha 
(2000, p. 1), pode ser entendida como "uma coleção organizada de dados multimídia com métodos de gerenciamento da informação, que representa os dados como informação útil e conhecimento para o povo numa variedade de contextos sociais e organizacionais".

Em consonância com os objetivos delineados na construção da Biblioteca Digital Paulo Freire, é possível entendê-la como um ambiente digital e multimídia que recepciona o conteúdo freireano, abrangendo seus pressupostos filosóficos, epistemológicos, sociológicos, culturais e pedagógicos rastreados nas diversas fontes de informação impressa e/ou eletrônica existentes, por meio do processo de busca, recuperação, armazenamento e disponibilização, com vistas à sua disseminação no mundo inteiro e a qualquer hora, possibilitando o acesso (www.paulofreire.ufpb.br), a interação e o uso por todos os cidadãos, independentemente de tempo, espaço, local, etnia, condição social e opção política.

O acervo físico dessa biblioteca está espalhado em vários espaços, demandando maior investimento nas etapas de busca, recuperação, seleção e armazenamento, disponibilização e dificultando a quantificação definitiva do acervo. Na sua primeira fase, foi possível localizar documentos em vários países, como Canadá, Suíça, Estados Unidos, México e França, e em vários estados brasileiros (Bezerra e Brennand, 2002).

Do ponto de vista das tecnologias utilizadas, as principais funções que concorrem para a criação e o funcionamento da Biblioteca Digital Paulo Freire - recuperação, digitalização e disponibilização de documentos e administração, distribuição e proteção de objetos multimídia - foram desenvolvidas por meio de exploração das potencialidades da tecnologia oferecida pelos seguintes softwares: IBM Digital Library, Lotus Notes, Microisis etc. Utilizamos equipamentos e softwares (plataforma de sistema operacional, definição de base de dados, software de OCR, software de edição de páginas, software de produção gráfica etc.) (Brennand et alii, 2000).

O formato digital estabelece uma ruptura com o modelo tradicional de biblioteca que a caracteriza como um espaço onde diversos itens do acervo são constituídos de documentos impressos. Essa biblioteca estabelece um link com as tecnologias da informação e comunicação, no qual o conceito de biblioteca se expande, podendose falar da biblioteca digital/virtual, em que a informação é armazenada de forma eletrônica e disseminada, independentemente de sua localização física ou de tempo.
A perspectiva de consolidação dessa biblioteca digital tem como eixo norteador o alargamento e a implementação de oportunidades de aprendizagem aberta e de participação dos cidadãos "info-pobres" no mundo da revolução digital que afeta, cada vez mais, a vida cotidiana da escola em seus aspectos globais, regionais e locais. Sua porta de entrada está aberta em qualquer momento, e o leitor, ao adentrá-la, poderá percorrê-la em todos os sentidos, saltando instantaneamente entre diferentes textos de Paulo Freire, dos autores que escreveram sobre esse educador para outros, de um suporte para outro e de uma temática para outra.

Esse formato de biblioteca não depende, nas suas aquisições, unicamente dos favores de mecenas, de esforços de bibliotecários ou de orçamentos de Estado, mas sobretudo do desejo de qualquer pessoa de publicar sobre um determinado autor o que entende que deva ser publicado (Fidalgo, 2001). Seja da sua autoria ou não, o seu acervo vai muito para além do produto da indústria editorial tradicional. Disponibilizar o conteúdo freireano "é compreender toda sua vida e obra, dedicada a interpretar e intervir nas práticas pedagógicas escolares e sociais, como uma ação cultural para a liberdade, [construindo] um pensamento que, ao pensar, se pensa a si mesmo" (Souza, 2001, p. 28).

\section{Artigo recebido em 27-08-2004 e aceito para publicação de} 18 a $21 / 10 / 2004$.

\section{REFERÊNCIAS}

AQUINO, Mirian de Albuquerque (Coord.). Recuperação do conteúdo Freireano para a construção da Biblioteca Digital Paulo Freire. João Pessoa : UFPB, 2001. (Projeto de iniciação científica. UFPB. Departamento de Biblioteconomia e Documentação).

BARTHES, Roland. O rumor da língua. Lisboa : Ed. 34, 1987.

BEZERRA, Ed Porto; BRENNAND, Edna G. Góes. Projeto implementação do pólo produtor de capacitação em conteúdos digitais multimídia no estado da Paraíba. João Pessoa : [S. n.], 2002.

BRENNAND, Edna G. Góes et al. Projeto concepção e implementação da Biblioteca Digital Paulo Freire. [S. 1. : s. n.], 2000.

CHEN, Ciang-Chih. As tecnologias multimídia. In: A INFORMAÇÃO: tendências para o novo milênio. [S. 1. : s. n.], 1999. 211 p.

CUNHA, Murilo Bastos da. Biblioteca digital: aspectos econômicos. Disponível em: <http:/www.ime.usp.Br/ cesar/simposio99/murilo/ sld001.htm>.

- Desafios na construção de uma biblioteca digital. Ciência da Informação, Brasília, v. 28, n. 3, p. 257-268, set./dez. 1999.

DOMINGUES, Diana. A humanização das tecnologias pela arte. In: . A ARTE no século XXI a humanização das tecnologias. São Paulo : UNESP, [2001?]. 


\section{Mirian de Albuquerque Aquino}

FIDALGO, Antônio. Disponível em: <http://bocc.ubi.pt/pag/fidalgobiblioteca.html>.

FREIRE, Paulo. Ação cultural para a liberdade e outros escritos. Rio de janeiro: Paz e Terra, 1982.

Pedagogia da indignação. São Paulo : UNESP, 2000.

GADOTTI, Moacir. As lições de Paulo Freire. In: PETER, M.; LEONARD, P; GADOTTI, Moacir (Org.). Paulo Freire: poder, desejo e memórias de libertação. Porto Alegre :ArtMed, 1998.

LÈVY, Pierre. A inteligência coletiva: por uma antropologia do ciberespaço. São Paulo : Loyola, 1994.
- As tecnologias da inteligência: o futuro do pensamento na era da informática. Rio de Janeiro : Ed. 34, 1996. 203 p.

$$
\text { Cibercultura. São Paulo : Ed. 34, } 1999 .
$$

MARTINS, Wilson. A palavra escrita: história do livro, da imprensa e da biblioteca. São Paulo : Ática, 1998.

PARENTE, André. O virtual e o hipertextual. Rio de Janeiro : Pazulin, 1999.

TAKAHASHI, Tadao (Org.). Sociedade da Informação no Brasil: livro verde. Brasília : Ministério da Ciência e Tecnologia, 2000.

SOUZA, Marco. Sala de aula interativa. Rio de Janeiro : Quarter, 2000. 